\title{
Automated CT Perfusion Prediction of Large Vessel Acute Stroke from Intracranial Atherosclerotic Disease
}

\author{
Diogo C. Haussen Mehdi Bouslama Seena Dehkharghani \\ Jonathan A. Grossberg Nicolas Bianchi Meredith Bowen \\ Michael R. Frankel Raul G. Nogueira \\ Emory University/Grady Memorial Hospital - Marcus Stroke and Neuroscience Center, \\ Atlanta, GA, USA
}

\author{
Keywords \\ Computed tomography $\cdot$ Stroke $\cdot$ Thrombectomy
}

\begin{abstract}
Background and Purpose: We have observed that large vessel occlusion acute strokes (LVOS) due to intracranial atherosclerotic disease (ICAD) present with more benign CT perfusion (CTP) profiles, which we presume to potentially represent enhanced collateralization compared to embolic LVOS. We aim to determine if CTP profiles can predict ICAD in LVOS. Methods: Retrospective review of a prospectively collected interventional stroke database from September 2010 to March 2015. Patients with intracranial ICA/MCA-M1/M2 occlusions and CTP were dichotomized into ICAD versus non-ICAD etiologies. Ischemic core (relative cerebral blood flow $<30 \%$ ) and hypoperfusion volumes were estimated by automated CTP. Results: A total of 250 patients met the inclusion criteria, comprised of 21 (8\%) ICAD and 229 non-ICAD etiologies. Baseline characteristics were similar between groups, except for higher $\mathrm{HbA1c}$ levels $(p<0.01)$, LDL cholesterol $(p<0.01)$, systolic blood pressure $(p<0.01)$, and lower rate of atrial fibrillation $(p<0.01)$ in ICAD patients. There were no significant differences in volumes of baseline ischemic core $(p=0.54)$ among groups. ICAD patients had smaller Tmax $>4 \mathrm{~s}$, Tmax $>6 \mathrm{~s}$, and Tmax $>10 \mathrm{~s}$ absolute lesions, and a higher ratio of Tmax $>4 \mathrm{~s} / \mathrm{Tmax}>6 \mathrm{~s}$ volumes (median 2 [1.6-2.3] vs. 1.6 [1.4-2.0]; $p=0.02$ ). A Tmax $>4 \mathrm{~s} / \mathrm{Tmax}>6$ s ratio $\geq 2$ showed specificity $=73 \%$ /sensitivity $=52 \%$ for ICAD and was observed in $47.6 \%$ of ICAD versus $26.1 \%$ of non-ICAD patients $(p=0.07)$. Clinical outcomes were comparable amongst groups. Multivariate logistic regression revealed that $\operatorname{Tmax}>4 \mathrm{~s} / \mathrm{Tmax}>6$ s ratio $\geq 2$ (OR 3.75, 95\% Cl 1.05$13.14, p=0.04)$, higher LDL cholesterol (OR 1.1, 95\% Cl 1.01-1.03, $p=0.01$ ), and higher sys-
\end{abstract}


tolic pressure (OR $1.03,95 \% \mathrm{Cl} 1.01-1.04, p=0.01)$ were independently associated with ICAD. Conclusion: An automated CTP Tmax $>4 \mathrm{~s} / \mathrm{Tmax}>6 \mathrm{~s}$ ratio $\geq 2$ profile was found independently associated with underlying ICAD LVOS.

(C) 2018 S. Karger AG, Basel

\section{Introduction}

Cerebral thrombectomy is technically challenging in patients with underlying intracranial atherosclerotic disease (ICAD) [1]. Unfortunately, there are no established biomarkers for ICAD and the lack of an adequate surrogate on noninvasive imaging makes procedural planning challenging. We have anecdotally observed that patients with large vessel occlusion acute stroke (LVOS) due to ICAD have more benign automated CT perfusion (CTP) profiles, which we presume may potentially reflect greater, chronic collateralization recruitment in comparison to embolic LVOS. We aim to determine if signature profiles on baseline CTP can predict the presence of underlying ICAD in LVOS.

\section{Methods}

\section{Patient Selection}

This was a retrospective review of a prospectively collected database of acute LVOS undergoing endovascular therapy spanning September 2010 to March 2015. Patients with a technically adequate CTP and an intracranial ICA, MCA-M1 or M2 occlusion were included. Tandem extracranial carotid occlusions and intracranial occlusions were excluded to minimize the delay and dispersion effects of extracranial steno-occlusive lesions upon dynamic bolus passage [2].

\section{Imaging}

CTP was post-processed in a fully automated, commercially available software environment (RAPID version 4.5.0, iSchemaView, CA, USA). Two contiguous CTP slabs were obtained for $8 \mathrm{~cm}$ of combined coverage of the supratentorial brain, obtained at 8 five-millimeter slices per slab. Cine mode acquisition (kV 80, auto-mA 100 ) permitting high temporal resolution (60 s sampling window and continuous $1 \mathrm{~s}$ sampling interval) dynamic bolus passage imaging was obtained following the administration of iodinated contrast. The total hypoperfused tissue volume was defined by the utilization of varying thresholds. A threshold of $>4 \mathrm{~s}$ delay for the maximum of the tissue residue function (Tmax) has historically defined tissues with likely benign oligemic delay, whereas ischemic territories with "tissue at risk" and "malignant hypoperfusion" have been assigned Tmax $>6 \mathrm{~s}$ and Tmax $>10 \mathrm{~s}$ thresholds, respectively [3]. The irreversible ischemic core volume was defined by a cerebral blood flow reduction to $<30 \%$ of the corresponding contralateral territory (relative cerebral blood flow $<30 \%$ ). Processed color parametric maps were overlaid upon source CTP data for review purposes [4].

Presumed ICAD etiology was defined by the presence of a fixed underlying stenosis (Arterial Occlusive Lesion score $=2$ ) observed after mechanical thrombectomy at the original occlusion site that had the typical contours of atherosclerotic plaques and did not respond to intra-arterial vasodilator infusion on delayed angiographic runs. For cases in which there was reocclusion or in which the underlying stenosis was flowlimiting, angioplasty/stent were typically performed. Cases of intracranial dissection (underlying or iatrogenic) and cases of vasospasm induced by the device were excluded.

Follow-up in all patients included MRI or CT documenting final infarct volumes before hospital discharge. Final infarct volumes were defined preferentially and predominantly by MRI. Final infarct volumes were measured using a manual segmentation tool, as previously reported [5]. This study was approved by the Institutional Review Board.

\section{Primary Analysis}

We have observed that patients with ICAD-related LVOS have larger proportional area of Tmax $>4 \mathrm{~s}$ (indicated by blue in the automated RAPID CTP maps) as compared to Tmax $>6$ s (indicated by green) (Fig. 1) as compared to non-ICAD strokes. This may relate to the chronic recruitment of more robust leptomeningeal 
Fig. 1. Illustrative case of a 54-year-old woman who developed stroke symptoms (NIHSS 29) and was confirmed to have intracranial atherosclerotic disease etiology. a Noninvasive imaging studies encompassed a CT angiogram at baseline revealing proximal left middle cerebral artery occlusion (left), automated CT perfusion with Tmax $>4 \mathrm{~s} /$ Tmax $>6 \mathrm{~s}$ ratio $>2$ (center), and final infarct by diffusion-weighted imaging (right). b Angiogram study confirming the occlusion (left), recanalization after one pass of stent retriever and underlying stenosis (middle), angioplasty and stenting (inset), and final angiogram (right).

Haussen et al.: Automated CT Perfusion Prediction of Large Vessel Acute Stroke from Intracranial Atherosclerotic Disease
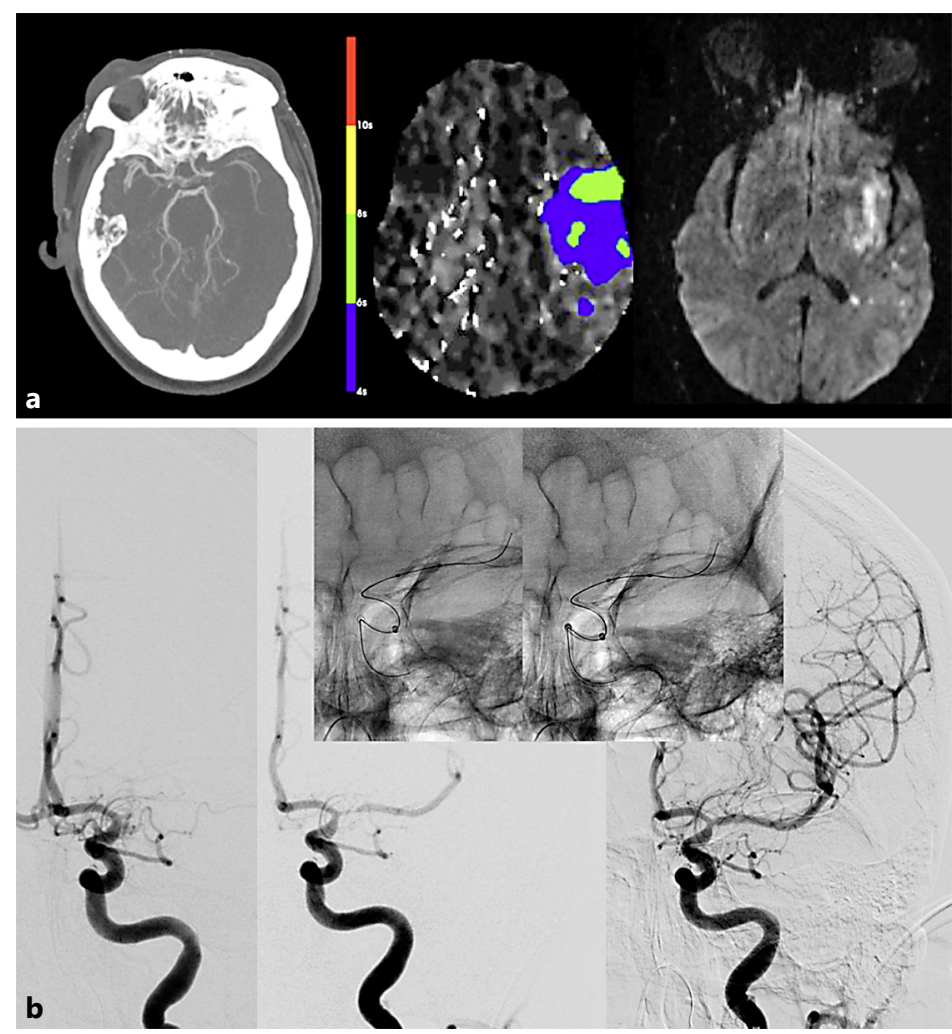

collateral flow/ischemic preconditioning [6]. Therefore, the primary endpoint was to stratify hypoperfusion profiles based upon the relative volumes of hypoperfused tissues defined at varying Tmax delays. Various ratios of hypoperfusion volumes were analyzed to identify signature profiles that may characterize the presence of preexisting, underlying ICAD. CTP Tmax $>4 \mathrm{~s} /$ Tmax $>6$ s volume ratios were examined for the ability to independently predict ICAD over other stroke subtypes.

\section{Statistical Analysis}

Accuracy was initially determined for different thresholds of the Tmax $>4 \mathrm{~s} / \mathrm{Tmax}>6 \mathrm{~s}$ ratio via receiver operating characteristic curve analysis, and the more accurate cutoff was utilized subsequently. Continuous variables are reported as mean $\pm \mathrm{SD} /$ median (IQR). Categorical variables are reported as proportions. Between groups, comparisons for continuous/ordinal variables were made with Student $t$ test, MannWhitney U, or analysis of variance, as appropriate. Categorical variables were compared by $\chi^{2}$ or Fisher exact test as appropriate. Significance was set at $p<0.05$. Multivariate logistic regression analysis for predictors of ICAD were performed for variables $<0.1$ level of significance (Backward-LR variable selection method). Statistical analyses were performed using SPSS $^{\circledR}$ Statistics $24\left(\right.$ IBM $^{\circledR}$, Armonk, NY, USA).

\section{Results}

A total of 250 patients fit the inclusion criteria, among whom 21 (8\%) were identified as having ICAD, while 229 (92\%) were classified as non-ICAD etiologies. Baseline characteristics were comparable between the two groups, except for higher levels of hemoglobin A1c $(p<$ $0.01)$, LDL cholesterol ( $p<0.01)$, and baseline systolic blood pressure $(p<0.01)$ as well as lower rates of atrial fibrillation $(p<0.01)$ in the ICAD group (Table 1$)$. Nine patients had intracranial stents placed in the ICAD group ( 5 balloon-mounted and 4 self-expanding) and one 
Table 1. Univariate analyses comparing the ICAD versus non-ICAD groups

\begin{tabular}{|c|c|c|c|}
\hline & $\operatorname{ICAD}(n=21)$ & Non-ICAD $(n=229)$ & $p$ value \\
\hline \multicolumn{4}{|l|}{ Baseline } \\
\hline Age & $66.5(61-76)$ & $65(51-76)$ & 0.99 \\
\hline Gender (male) & $8(38 \%)$ & $103(45 \%)$ & 0.64 \\
\hline Hypertension & $18(85 \%)$ & $151(66 \%)$ & 0.06 \\
\hline Hyperlipidemia & $7(33 \%)$ & $64(28 \%)$ & 0.61 \\
\hline LDL cholesterol & $119(88-148)$ & $80(60.5-104)$ & $<0.001$ \\
\hline Diabetes & $11(52 \%)$ & $49(21 \%)$ & $<0.01$ \\
\hline Hemoglobin A1c & $6(5.6-7.6)$ & $5.7(5.3-6.1)$ & 0.01 \\
\hline Glucose & $126(113-313)$ & $119(102-135)$ & 0.55 \\
\hline Atrial fibrillation & $2(9 \%)$ & $93(40 \%)$ & $<0.01$ \\
\hline Smoking & $4(19 \%)$ & $30(13 \%)$ & 0.44 \\
\hline Systolic blood pressure & $175(150-200)$ & $142(127-165)$ & $<0.001$ \\
\hline NIHSS & $13.5(8-19)$ & $18(12-22)$ & 0.09 \\
\hline IV t-PA & 7 (35\%) & $93(40.8 \%)$ & 0.81 \\
\hline Last-normal to puncture & $444(336-514)$ & $340(236-545)$ & 0.43 \\
\hline \multicolumn{4}{|l|}{ Noninvasive imaging } \\
\hline ASPECTS & $8(7-10)$ & $8(7-9)$ & 0.08 \\
\hline Relative cerebral blood flow $<30$ & $8(0-20)$ & $7(0-23)$ & 0.54 \\
\hline $\operatorname{Tmax}>4 \mathrm{~s}$ & $181(145-224)$ & $224(167-308)$ & 0.02 \\
\hline $\operatorname{Tmax}>6 \mathrm{~s}$ & $83(62-162)$ & $137(84-185)$ & $<0.01$ \\
\hline $\operatorname{Tmax}>10 \mathrm{~s}$ & $21(8-87)$ & $66(21-105)$ & 0.03 \\
\hline $\operatorname{Tmax}>4 \mathrm{~s}-\mathrm{Tmax}>6 \mathrm{~s}$ & $86(60-137)$ & $85(60-131)$ & 0.79 \\
\hline $\operatorname{Tmax}>4 \mathrm{~s} / \operatorname{Tmax}>6 \mathrm{~s}$ & $2(1.6-2.3)$ & $1.6(1.4-2.0)$ & 0.02 \\
\hline Tmax $>4$ s/Tmax $>6$ s (ratio $\geq 2$ ) & $10(47.6 \%)$ & $62(27.1 \%)$ & 0.07 \\
\hline \multicolumn{4}{|l|}{ Procedure } \\
\hline Procedure length & $95.5(45-127)$ & $62(39-91)$ & 0.70 \\
\hline Baseline mTICI 1-2A & $2(9.5 \%)$ & $9(3.9 \%)$ & 0.23 \\
\hline Conscious sedation & $16(76.2 \%)$ & $188(82.1 \%)$ & 0.56 \\
\hline Stent retriever & $17(81 \%)$ & $176(76.9 \%)$ & 0.79 \\
\hline Intracranial stent & $9(42.8 \%)$ & $2(0.9 \%)$ & $<0.001$ \\
\hline \multicolumn{4}{|l|}{ Outcomes } \\
\hline mTICI $2 b-3$ reperfusion & $20(95.2 \%)$ & 207 (90.4\%) & 0.70 \\
\hline mTICI 3 reperfusion & $13(61.9 \%)$ & $107(46.7 \%)$ & 0.25 \\
\hline Parenchymal hemorrhage & $0(0 \%)$ & $20(8.7 \%)$ & 0.39 \\
\hline 90-day mRS 0-2 & $9(47.4 \%)$ & $115(55 \%)$ & 0.63 \\
\hline 90-day mortality & $3(15.8 \%)$ & $32(15.3 \%)$ & 1.00 \\
\hline Final infarct volumes, $\mathrm{mL}$ & $21(5-43)$ & $21(8-48)$ & 0.10 \\
\hline
\end{tabular}

ASPECTS, Alberta Stroke Program Early CT score; LDL, low-density lipoprotein; mTICI, modified Thrombolysis in Cerebral Infarction; NIHSS, National Institutes of Health Stroke Scale; mRS, modified Rankin Scale.

had intracranial angioplasty only. Reperfusion rates and clinical outcomes were comparable between groups.

Analysis of presentation on CTP revealed no significant differences in the baseline volumes of ischemic core among patients with and without ICAD (8.0 cc vs. $7.0 \mathrm{cc} ; p=0.54$ ). Patients with ICAD had a more benign perfusion defect profile with smaller Tmax $>4 \mathrm{~s}$, Tmax $>6 \mathrm{~s}$, and Tmax $>10 \mathrm{~s}$ (Table 1). The ratio of Tmax $>4 \mathrm{~s} / \mathrm{Tmax}>6 \mathrm{~s}$ volumes was higher in the ICAD (median 2 [1.6-2.3]) versus non-ICAD (1.6 [1.4-2.0]) group ( $p=0.02)$. A Tmax $>4 \mathrm{~s} /$ Tmax $>6$ s ratio $\geq 2$ was observed in $47.6 \%$ of the ICAD versus $26.1 \%$ of the non-ICAD patients 
Table 2. Multivariable logistic regression for predictors of ICAD etiology

\begin{tabular}{llll}
\hline & OR & $95 \% \mathrm{CI}$ & $p$ value \\
\hline LDL cholesterol & 1.01 & $1.01-1.03$ & 0.01 \\
Systolic blood pressure & 1.03 & $1.01-1.04$ & 0.01 \\
CTP Tmax $>4$ s/Tmax $>6$ s ratio $\geq 2$ & 3.75 & $1.05-13.14$ & 0.04 \\
Atrial fibrillation & 0.22 & $0.04-1.12$ & 0.06 \\
Hypertension & 7.18 & $0.73-70.19$ & 0.09 \\
NIHSS & 0.91 & $0.82-1.02$ & 0.10 \\
\hline
\end{tabular}

LDL, low-density lipoprotein; NIHSS, National Institutes of Health Stroke Scale.

( $p=0.07)$. A Tmax $>4 \mathrm{~s} / \mathrm{Tmax}>6 \mathrm{~s}$ ratio $\geq 2$ had an accuracy of $71 \%$, and as per the receiver operating characteristic curve analysis, $52 \%$ sensitivity and $73 \%$ specificity for the detection of ICAD.

Multivariate logistic regression analysis revealed that Tmax $>4 \mathrm{~s} / \mathrm{Tmax}>6 \mathrm{~s}$ ratio $\geq 2$ (OR 3.75, 95\% CI 1.05-13.14, $p=0.04$ ), higher LDL cholesterol levels (OR 1.1, 95\% CI 1.01-1.03, $p=0.01$ ), and higher systolic pressure (OR 1.03, 95\% CI 1.01-1.04, $p=0.01$ ) were independently associated with ICAD (Table 2).

\section{Discussion}

Our findings demonstrate that patients with ICAD have quantitatively and qualitatively enhanced collateral flow as per CTP analysis. Furthermore, specific patterns of hypoperfusion derived from CTP may be predictive of ICAD LVOS etiology in a large cohort of patients undergoing evaluation for LVOS.

Thrombectomy in ICAD patients is often more technically demanding. Procedure length has been described to be longer, reperfusion rate to be lower, and early reocclusions to be more common $[1,7]$. Early detection of ICAD facilitates avoidance of repeated stent retriever passes (resulting in less endothelial denudation), and earlier definitive revascularization maneuvers, such as angioplasty and stenting. Moreover, considering the tendency for early reocclusion, the time to heparinization and antiplatelet administration can be shortened [8].

A few studies have evaluated potential clinical predictors of ICAD. Atrial fibrillation has consistently been found to be inversely associated with ICAD, while hyperlipidemia and diabetes have been shown to be directly associated with it $[9,10]$. Our findings indicate ICAD LVOS patients have greater than twice the frequency of diabetes compared to non-ICAD, as well as higher hemoglobin A1c and LDL cholesterol levels. NIHSS was found to be nonstatistically lower in our ICAD cohort, which is in line with previous reports $[6,9,10]$. Such baseline characteristics are, however, ubiquitous within the LVOS population and are therefore not robust predictors of underlying ICAD etiology.

Radiological markers of ICAD have also been relatively nonspecific. Gradient echo, T2*weighted susceptibility defects in the occluded vessel on MRI has been demonstrated to be strongly associated with cardioembolic strokes. Conversely, cases of in situ thrombosis have been reported to present with relatively small superimposed thrombus burden [7]. However, among patients without such a susceptibility sign on T2*-weighted MRI, fewer than half were found to have ICAD [11].

Patients with ICAD may have better collateral recruitment as compared to other stroke subtypes due to ischemic preconditioning. Indeed, patients with in-situ thrombosis have 
been demonstrated to have better angiographic collaterals as compared to patients embolic LVOS [12]. In an Asian study of 86 patients evaluated with MR perfusion imaging, the severity of the perfusion defect was determined by a ratio of Tmax $\geq 8 \mathrm{~s} / \mathrm{Tmax} \geq 2 \mathrm{~s}$ volumes, and was found to be lower in ICAD as compared to other stroke subtypes [6]. Our study corroborates these observations and identified, after adjustment for covariates, a CTP pattern predictive of ICAD. Comparatively, we analyzed a homogeneous sample solely consisted of LVOS that underwent thrombectomy and utilized established thresholds for tissue at risk [4]. We have shown that an automated CTP profile exhibiting a disproportionately large volume of Tmax $>4 \mathrm{~s}$ tissues relative to Tmax $>6 \mathrm{~s}$ volume may favor the presence of an LVOS with underlying ICAD. This finding may constitute a useful predictor of the presence of an atherosclerotic steno-occlusive lesion with in situ thrombosis.

This study has several limitations, including those directly inherent to the retrospective nature. Despite the modest sample size, this represents to our knowledge the largest study of CTP in the evaluation of LVOS due to ICAD. Despite conventional angiography being considered the gold standard for diagnosis of ICAD, the proposed definition might have introduced selection bias. The relatively low median ischemic core size of the investigated population may have introduced selection bias since patients with larger strokes could have different perfusion patterns. The lack of follow-up vascular imaging compromises the ability of evaluating for posttreatment angioarchitectural changes and reocclusions.

In summary, the automated CTP perfusion defect severity ratio of Tmax $>4 \mathrm{~s} / \mathrm{Tmax}>6 \mathrm{~s}$ $\geq 2$ profile is an independent indicator of underlying ICAD in LVOS. The value of this noninvasive imaging finding should be further investigated.

\section{Disclosure Statement}

D.C.H./M.B./J.A.G./N.B./M.B./M.R.F.: none. R.G.N.: Stryker (PI:Trevo-2 PI/DAWN Trials), Covidien (SWIFT/SWIFT-PRIME Steering Committee, STAR Trial Core-Lab), and Penumbra (3-D Trial Executive Committee).

\section{References}

1 Al Kasab S, Almadidy Z, Spiotta AM, Turk AS, Chaudry MI, Hungerford JP, Turner RDt: Endovascular treatment for AIS with underlying ICAD. J Neurointerv Surg 2017;9:948-951.

-2 Haussen DC DS, Grigoryan M, Bowen M, Rebello LC, Nogueira RG: Automated CT perfusion for ischemic core prediction in anterior circulation tandem occlusions. Intervent Neurol 2016;5:81-88.

-3 Lansberg MG, Straka M, Kemp S, Mlynash M, Wechsler LR, Jovin TG, Wilder MJ, Lutsep HL, Czartoski TJ, Bernstein RA, Chang CW, Warach S, Fazekas F, Inoue M, Tipirneni A, Hamilton SA, Zaharchuk G, Marks MP, Bammer R, Albers GW; DEFUSE 2 study investigators: MRI profile and response to endovascular reperfusion after stroke (DEFUSE 2): a prospective cohort study. Lancet Neurol 2012;11:860-867.

4 Albers GW, Goyal M, Jahan R, Bonafe A, Diener HC, Levy EI, Pereira VM, Cognard C, Cohen DJ, Hacke W, Jansen O, Jovin TG, Mattle HP, Nogueira RG, Siddiqui AH, Yavagal DR, Baxter BW, Devlin TG, Lopes DK, Reddy VK, du Mesnil de Rochemont R, Singer OC, Bammer R, Saver JL: Ischemic core and hypoperfusion volumes predict infarct size in SWIFT PRIME. Ann Neurol 2016;79:76-89.

5 Dehkharghani S, Bammer R, Straka M, Albin LS, Kass-Hout O, Allen JW, Rangaraju S, Qiu D, Winningham MJ, Nahab F: Performance and predictive value of a user-independent platform for CT perfusion analysis: threshold-derived automated systems outperform examiner-driven approaches in outcome prediction of acute ischemic stroke. AJNR Am J Neuroradiol 2015;36:1419-1425.

6 Kim SJ, Seok JM, Bang OY, Kim GM, Kim KH, Jeon P, Chung CS, Lee KH, Alger JR, Liebeskind DS: MR mismatch profiles in patients with intracranial atherosclerotic stroke: a comprehensive approach comparing stroke subtypes. J Cereb Blood Flow Metab 2009;29:1138-1145.

7 Lee JS, Hong JM, Lee KS, Suh HI, Choi JW, Kim SY: Primary stent retrieval for acute intracranial large artery occlusion due to atherosclerotic disease. J Stroke 2016;18:96-101.

8 Haussen DC, Liebeskind DS, Smith W, Lutsep H, Jovin T, Xiang B, Nogueira RG: Peri-procedural heparin use in stent-retriever thrombectomy. Stroke 2016;47:AWP10. 
9 Yoon W, Kim SK, Park MS, Kim BC, Kang HK: Endovascular treatment and the outcomes of atherosclerotic intracranial stenosis in patients with hyperacute stroke. Neurosurgery 2015;76:680-686; discussion 686.

10 Lee JS, Hong JM, Lee KS, Suh HI, Demchuk AM, Hwang YH, Kim BM, Kim JS: Endovascular therapy of cerebral arterial occlusions: intracranial atherosclerosis versus embolism. J Stroke Cerebrovasc Dis 2015;24:20742080.

11 Cho KH, Kim JS, Kwon SU, Cho AH, Kang DW: Significance of susceptibility vessel sign on T2*-weighted gradient echo imaging for identification of stroke subtypes. Stroke 2005;36:2379-2383.

12 Hwang YH, Kim YW, Kang DH, Kim YS, Liebeskind DS: Impact of target arterial residual stenosis on outcome after endovascular revascularization. Stroke 2016;47:1850-1857. 